\title{
Visual neglect
}

\section{Paolo Bartolomeo}

Inserm U 610, Hôpital de la Salpêtrière, Paris, France; Fédération de Neurologie, AP-HP, Hôpital de la Salpêtrière, Paris, France; Université Pierre et Marie Curie-Paris6, Paris, France

Address correspondence to:

Paolo Bartolomeo

INSERM U 610

Pavillon Claude Bernard

Hôpital Salpêtrière

47 bd de l'Hôpital

F-75013 Paris - France

phone $+33(0) 142160025$ or 58

FAX +33 (0) 142164195

Email: paolo.bartolomeo@chups.jussieu.fr

Web: http://marsicanus.free.fr/ 


\section{Purpose of review}

Left visual neglect is a frequent and dramatic consequence of right hemisphere lesions. Diagnosis is important because behavioural and pharmacological treatments are available. Furthermore, neglect raises important issues concerning the brain mechanisms of consciousness, perception and attention.

\section{Recent findings}

Recent behavioural findings and new techniques, such as transcranial magnetic stimulation, direct cortical and subcortical stimulation during brain surgery, and diffusion tensor imaging tractography, have provided evidence relevant to the debate concerning the functional mechanisms and the anatomical bases of neglect.

\section{Summary}

Several component deficits appear to interact in producing different forms of neglect. Rather than lesions at single cortical levels, dysfunction of large-scale brain networks, often induced by white matter disconnection, may constitute the crucial antecedent of neglect signs.

\section{Keywords}

Spatial cognition, Perception, Attention, Mental imagery, White matter fiber pathways 


\section{Abbreviations}

$\begin{array}{ll}\text { BA } & \text { Brodmann area } \\ \text { CT } & \text { computerised tomography } \\ \text { DT } & \text { diffusion tensor } \\ \text { FEF } & \text { frontal eye field } \\ \text { IPL } & \text { inferior parietal lobule } \\ \text { MRI } & \text { magnetic resonance imaging } \\ \text { REMs } & \text { rapid eye movements } \\ \text { SLF } & \text { superior longitudinal fasciculus } \\ \text { STG } & \text { superior temporal gyrus } \\ \text { TMS } & \text { transcranial magnetic stimulation }\end{array}$




\section{Introduction}

In a neurological ward, it is frequent to come upon patients who look at objects on their right side with intense interest, while paying no attention to what happens on their left. Visual neglect is a dramatic but often overlooked consequence of right hemisphere damage, usually of vascular origin. Patients do not eat from the left part of their dish, they bump their wheelchair into obstacles situated on their left, and have a tendency to look to right-sided details as soon as a visual scene deploys, as if their attention were 'magnetically' attracted by these details [1]. They are usually unaware of their deficits (anosognosia), and often obstinately deny being hemiplegic [2]. Patients with left brain damage may also show signs of contralesional, right-sided neglect, albeit more rarely and usually in a less severe form [3]. Diagnosis is important, because neglect predicts poor functional outcome in stroke [4]. Moreover, effective rehabilitation strategies are available [5], and there are promising possibilities for pharmacological treatments [6].

\section{Bedside testing}

A few paper-and-pencil tests, which can be administered at bedside, can confirm diagnosis [7]. Neglect patients omit to cancel left targets in search tasks, deviate rightward when bisecting horizontal lines, fail to copy the left part of drawings [8].

Patients' asymmetries of performance in cancellation tasks scan vary from a few left-sided omissions, to cancellation of only the rightmost items. Some patients will cancel again and again the same right-sided items, thus showing a pathological "revisiting behaviour" for objects presented in the supposedly "normal" sector of space [9]. Patients who can compensate for their deficit to some extent, either as a result of spontaneous recovery or after rehabilitation, may cancel out all the elements, but keep starting from the right extremity of the sheet, at variance with normal participants, who most often start from the left part of the sheet [10], perhaps as a consequence of the left-to-right reading habits typical of Western cultures (see 
[11]).

Line bisection is also a useful tool to discriminate between neglect and visual field defects, such as left homonymous hemianopia, which was once thought to cause neglect. Contrary to this hypothesis, there are patients with left hemianopia but no neglect, who deviate leftward on line bisection $[12,13,14]$. The association of left neglect and hemianopia, however, produces the largest rightward deviations on line bisection $[12,13,15]$. When given relatively short lines to bisect (e.g., $5 \mathrm{~cm}$ or less), patients may paradoxically shift the bisection point leftwards (so-called crossover effect) [16]. The co-presence of visual field defects may be a necessary condition for this [17] and other neglect-related behaviours [15] to occur.

When copying a drawing, neglect patients often omit left-sided details (more rarely, patients may increase the number and spatial extension of left-sided details [18*]). When drawing well-known objects from memory, patients may demonstrate similar omissions of left-sided details. Surprisingly, however, some of these patients make more symmetrical drawings when blindfolded than in free vision (Fig. 1) [19]. Thus, even in drawing from memory, patients' attention may be "magnetically" drawn to the right-sided details they just drew, rendering difficult the completion of the drawing on the left, neglected side. Perhaps sensory deprivation might be used in neglect rehabilitation to offset the attention-capturing power of right-sided visual details.

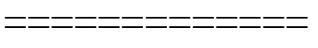

Fig. 1 about here

\section{Imaginal neglect}

Despite these demonstrations of the importance of right-sided visual stimuli in eliciting left neglect, neglect can manifest itself in the absence of visual stimuli. When describing known places from memory, patients 
may omit details situated in the left part of the (mental) scene [20]. However, only a minority of patients with visual neglect also show imaginal neglect, perhaps because imagined details have less attention-capturing power than real ones [10,21]. Imaginal neglect can also occur in the absence of signs of perceptual neglect, either at onset [22] or, perhaps more commonly, as a result of selective compensation for the perceptual aspects of the syndrome [10]. Patients often learn with time (and possibly the help of people around them) to explore more thoroughly their visual environment. However, compensation may be more difficult to obtain in the more abstract imaginal domain, which is rarely the object of rehabilitation or of more informal reminders to "look to your left".

Neglect patients may also deviate rightwards on the mental bisection of number intervals; for example, when asked which is the median number between 11 and 19 , they may answer " 17 " [23*]. In this domain as well, visual and imaginal performance may dissociate. Biased performance with mental number lines might be related to concomitant prefrontal damage and spatial working memory impairment [24]. It would indeed be surprising to find that all neglect patients demonstrate such a mental bias, given that people do not always imagine numbers in spatial arrangements, and even when they do, their mental diagrams are not necessarily oriented along the horizontal direction [25].

When a patient was asked to use a black touch screen to represent the night sky, and to touch the locations occupied by (imaginary) stars [26*], he put significantly more stars to the right of the screen midline, but especially when the stars remained illuminated after the touch. If the screen remained black, the asymmetry was less evident. This again suggests an attention-capturing influence of real right-sided visual stimuli on patients' neglect [19,27]. But perceptual influences on spatial imagery seem less relevant for casual, task-unrelated stimuli. When patients were asked to imagine and describe the map of France with eyes open or blindfolded, performance was similar regardless of the condition [28*]. During sleep, neglect patients may show suppression of leftward-directed REMs. A recent case report described a patient with left visual neglect and frequent nystagmoid REMs with alternating leftward slow/rightward fast phases, 
corresponding to dreams with consistent visual events, such as a train running leftward, but virtually no nystagmoid REMs in the opposite direction [29**]. The complex relationships between perception and imagery in general [30], and concerning neglect in particular, are difficult to predict from the available theoretical models.

\section{Functional mechanisms}

It should be clear even from this brief description that left neglect cannot be considered as a unitary, homogeneous entity. Several dissociations of performance have been described between the outcomes of neuropsychological tasks, whether clinical [31] or experimental [32*]. It has proven difficult to find a clear correspondence between behavioural dissociations and different lesion localizations, perhaps because clinical, low-definition images are often used, and the focus has mainly been on grey matter lesions (see below). As mentioned before, a possible source of (spurious) dissociations may result from patients learning to use compensatory strategies in a domain but not in another. This occurrence may be difficult or impossible to ascertain; functional dissociations with corresponding lesional differences (e.g., [24,31]) seem the best suited to substantiate claims for different underlying causes.

The possible mechanisms leading to neglect have fostered considerable debate during the last decades. Several independent deficits, probably interacting with each other, may contribute to neglect signs. These may include deficits in orienting of spatial attention [33], in building or maintaining spatial representations [34], or in programming left-directed hand movements [35*]. It is also possible, however, than some deficits have more weight than others in shaping patients' behaviour. For example, deficits of spatial attention, such as an engagement of attention towards right-sided, non-neglected items as soon as the visual scene unfolds $[1,32 *, 36,37 *]$, followed by impaired disengagement from these same items [38], have

often been considered key component deficits of neglect. Importantly, these deficits seem mainly to concern exogenous, or stimulus-related, orienting of attention, with relative sparing of endogenous, or voluntary, 
orienting $\left[32^{*}, 39\right]$. Thus, the simple presence of right-sided distractors can disrupt patients' performance $[27,40 * *]$. Also non-lateralized deficits can contribute, perhaps crucially [41,42], to clinical neglect. For example, also processing of items presented in central [43] or right-sided locations $[44,45]$ can be impaired in left neglect. However, attentional deficits can occur after right brain damage even in the absence of clinical neglect $[38,46,47 *]$, consistent with the idea that several deficits must combine to produce overt neglect behaviour $\left[1,35^{*}\right]$.

\section{Lesional correlates}

In keeping with the multifarious nature of their symptoms, patients with neglect often have relatively large lesions of the right hemisphere, which are likely to disrupt several functional modules. However, the precise localization of these lesions still remains controversial. Neglect patients' lesions, as detected by CT or MRI, often overlap on the inferior parietal lobule (IPL), at the junction with the temporal lobe [48]. Conflicting evidence, however, also indicates lesions of the middle and rostral parts of superior temporal gyrus (STG) $\left[49^{*}, 50\right]$, and tends to exclude a role for lesions of the temporo-parietal junction [51]. Recent proposals have suggested that parietal or STG dysfunction may lead to different forms of neglect (respectively, personal/extrapersonal [49*], or viewer-centred/stimulus centred [52]). However, the lesion overlap method obviously lacks spatial resolution, may reflect differences in vascular territories rather than true functional architecture, and does not satisfactorily deal with multiple lesions [53,54]. Thus, other neuroimaging techniques have recently been applied to the study of the neural bases of neglect.

Transcranial magnetic stimulation (TMS) transiently disrupts the integrated activity of cortical networks in a relatively non-invasive fashion. TMS over the left hemisphere decreased left extinction and neglect in right brain-damaged patients [55]. Temporary inactivation of the middle/rostral portions of the STG produced non-lateralized impairments in visual search tasks [56]. In the same study, TMS stimulation of the central sectors of the STG did not modify judgments of the length of horizontal lines (Landmark task), in 
contrast to inactivation of the posterior parietal cortex, which provoked lateralized effects similar to that shown by patients with neglect on the same task.

Functional MRI has been employed to explore the neural correlates of sub-acute and recovered neglect [57]. Four weeks after a stroke, when performing a response time task to lateralized stimuli, neglect patients had decreased activation of structurally intact fronto-parietal regions in the right hemisphere (especially the intraparietal sulcus, the superior parietal lobule and the dorsolateral prefrontal cortex), coupled with robust activation of the homologous regions in the left hemisphere. Thirty-nine weeks after lesion onset, recovery of neglect signs was paralleled by the disappearance of the imbalance between the two superior parietal lobules. Thus, lesions of the right temporo-parietal junction may determine a functional imbalance of the superior parietal lobules, which are structures important to attentional orienting, with consequent biased orienting towards right-sided objects. A promising implication of these results is the possibility to ameliorate left neglect by functionally inhibiting the left parietal lobe using TMS [55].

Temporary electrical inactivation of small brain regions $(\sim 5 \mathrm{~mm})$ stimuli can be performed during brain surgery to prevent post-operative deficits. Thiebaut de Schotten et al [58] described the performance of two patients who bisected horizontal lines while being submitted to the surgical resection of low-grade gliomas. Patients deviated rightward upon inactivation of the supra-marginal gyrus (the rostral subdivision of IPL) and of the caudal part of the STG; however, bisection performance was accurate when more rostral portions of the STG or the frontal eye field (FEF) were inactivated. These findings run counter to a strong version of the STG hypothesis [51], at least as far as line bisection is concerned. Importantly, however, the strongest deviations occurred in one patient upon inactivation of a white-matter region in the depth of the IPL after most of the tumour had been removed. The course of long association fibres in the white matter of this particular patient was mapped in post-operative MRI scans using diffusion tensor MRI tractography (DTMRI), a new technique capable of tracking white matter fibres [59*]. The tract whose inactivation had brought about the maximal rightward deviation corresponded to the likely human homologous of the second 
branch of the superior longitudinal fasciculus [60**]. This pathway connects the inferior and the superior parietal lobules, particularly the angular gyrus (BA 39), including the intraparietal sulcus (IPS), to the middle and superior frontal gyri (BA 9, 8, 46 and 6) [61]. The observation that functional fronto-parietal disconnection dramatically disrupted the symmetrical processing of the visual scene is consistent with many findings obtained in rodents, in non-human primates and in human stroke patients (see [54,62**], for review). A more recent intraoperative stimulation study [63] confirmed the TMS findings reviewed above [56], by showing that electrical inactivation of the central STG during brain surgery produced non-lateralised impairments in visual search. These results $[56,63]$ thus seem consistent with the possibility that regions of the right temporal lobe are important for visual recognition and memory [64], but their relevance to neglect remains unclear.

DT-MRI tractography can be used to track the long-range white matter pathways (Fig. 2) and then explore, in a standardized brain space, their relationships with the lesions found in stroke patients with standard, anatomical MRI. Thus, for the first time white matter pathways can be explored in detail in the living human brain, and the focus can shift from impairment of cortical modules to dysfunction of cortical networks [59*]. A recent meta-analysis [62**] of previous lesion overlapping studies demonstrated that the subcortical lesions of neglect patients invariably overlapped at or near the human homologues of SLF II and III. Disconnection between cortical modules might thus be a general mechanism of neglect [54]. This possibility is also consistent with the results of computer simulations of attention [65*].

\section{$=========$}

Fig. 2 about here

These results support models of neglect which postulate a dysfunction of large-scale right-hemisphere networks [66]. Parietal components of the network could determine the perceptual salience of extrapersonal 
objects; frontal components might be implicated in the production of an appropriate response to behaviorally relevant stimuli, in the online retention of spatial information, or in the focusing of attention on salient items through reciprocal connections to more posterior regions. The network approach might prove important for patient diagnosis because a particular form of white matter disconnection might have greater predictive value than the localization of grey matter lesions. The demonstration of anatomically intact but functionally inactivated areas might also open perspectives for treatments (whether pharmacological or rehabilitative), aimed at restoring normal neural activity in these areas.

Although neglect commonly results from lesions in the territory of the middle cerebral artery, posterior cerebral artery strokes can also give rise to neglect signs. Also in these patients, the presence of neglect seems to correlate with inter-and intrahemispheric disconnection [67*]. Bird et al. [68*] located the maximal lesion overlap on a white matter tract linking the parahyppocampal gyrus to the angular gyrus, as tracked using DT-MRI of a normal individual.

\section{Conclusion}

Neglect remains a highly controversial topic, both concerning its mechanisms and its neural bases. Besides its clinical importance, its study has implications for our understanding of attention, consciousness, and perception. Research on the functional mechanisms appears to be moving from the description of dissociations in patients' performance to the dissection of the possible component deficits and of their modes of interaction. New, high-resolution imaging techniques are providing evidence relevant to the debate on the anatomical bases of neglect, shifting the focus from the study of cortical modules to large-scale brain networks. A huge explanatory gap still separates the functional and the anatomical descriptions of neglect, but it is a gap which seems now to be narrowing at a fast pace. 


\section{References}

1 Gainotti G, D'Erme P, Bartolomeo P: Early orientation of attention toward the half space ipsilateral to the lesion in patients with unilateral brain damage. Journal of Neurology, Neurosurgery and Psychiatry 1991, 54:1082-1089.

2 Berti A, Bottini G, Gandola M, Pia L, Smania N, Stracciari A, Castiglioni I, Vallar G, Paulesu E: Shared cortical anatomy for motor awareness and motor control. Science 2005, 309:488-491.

3 Beis JM, Keller C, Morin N, Bartolomeo P, Bernati T, Chokron S, Leclercq M, Louis-Dreyfus A, Marchal $F$, Martin $Y$, et al.: Right spatial neglect after left hemisphere stroke: Qualitative and quantitative study. Neurology 2004, 63:1600-1605.

4 Malhotra P, Coulthard E, Husain M: Hemispatial neglect, balance and eye-movement control. Current Opinion in Neurology 2006, 19:14-20.

5 Pisella L, Rode G, Farne A, Tilikete C, Rossetti Y: Prism adaptation in the rehabilitation of patients with visuo-spatial cognitive disorders. Current Opinion in Neurology 2006, 19:534-542.

6 Coulthard E, Singh-Curry V, Husain M: Treatment of attention deficits in neurological disorders. Current Opinion in Neurology 2006, 19:613-618.

7 Azouvi P, Bartolomeo P, Beis J-M, Perennou D, Pradat-Diehl P, Rousseaux M: A battery of tests for the quantitative assessment of unilateral neglect. Restorative Neurology and Neuroscience 2006, 24:273-285.

8 Bartolomeo $\mathrm{P}$, Chokron S: Levels of impairment in unilateral neglect. In Handbook of Neuropsychology, edn 2nd. Edited by Boller F, Grafman J: Elsevier Science Publishers; 2001:67-98. vol 4.]

9 Mannan SK, Mort DJ, Hodgson TL, Driver J, Kennard C, Husain M: Revisiting previously searched locations in visual neglect: role of right parietal and frontal lesions in misjudging old locations as new. Journal of Cognitive Neuroscience 2005, 17:340-354.

10 Bartolomeo P, D'Erme P, Gainotti G: The relationship between visuospatial and representational neglect. Neurology 1994, 44:1710-1714.

11 Chokron S, Bartolomeo P, Perenin MT, Helft G, Imbert M: Scanning direction and line bisection: A study of normal subjects and unilateral neglect patients with opposite reading habits. Cognitive Brain Research 1998, 7:173-178.

12 Bartolomeo P: Aspetti dell'emi-inattenzione spaziale nelle lesioni emisferiche: fattori che influenzano la bisezione di linee [Aspects of spatial hemi-inattention: factors influencing line bisection performance]. Unpublished MD thesis, Roma: Università Cattolica: 1987.

13 D'Erme P, De Bonis C, Gainotti G: Influenza dell'emi-inattenzione e dell'emianopsia sui compiti di 
bisezione di linee nei pazienti cerebrolesi [Influence of unilateral neglect and hemianopia on line bisection performance in brain-damaged patients]. Archivio di Psicologia, Neurologia e Psichiatria 1987, 48:165-189.

14 Barton JJ, Black SE: Line bisection in hemianopia. Journal of Neurology, Neurosurgery and Psychiatry 1998, 64:660-662.

15 Doricchi F, Angelelli P: Misrepresentation of horizontal space in left unilateral neglect: role of hemianopia. Neurology 1999, 52:1845-1852.

16 Marshall JC, Halligan PW: When right goes left: An investigation of line bisection in a case of visual neglect. Cortex 1989, 25:503-515.

17 Doricchi F, Guariglia P, Figliozzi F, Silvetti M, Bruno G, Gasparini M: Causes of cross-over in unilateral neglect: between-group comparisons, within-patient dissociations and eye movements. Brain 2005, 128:1386-1406.

* 18 Rode G, Michel C, Rossetti Y, Boisson D, Vallar G: Left size distortion (hyperschematia) after right brain damage. Neurology 2006, 67:1801-1808.

Two neglect patients showed a paradoxical enlargement of the left part of their drawings.

19 Chokron S, Colliot P, Bartolomeo P: The role of vision on spatial representations. Cortex 2004, 40:281-290.

20 Bisiach E, Luzzatti C: Unilateral neglect of representational space. Cortex 1978, 14:129-133.

21 Bartolomeo P, Bachoud-Lévi A-C, Azouvi P, Chokron S: Time to imagine space: a chronometric exploration of representational neglect. Neuropsychologia 2005, 43:1249-1257.

22 Ortigue S, Viaud-Delmon I, Annoni JM, Landis T, Michel C, Blanke O, Vuilleumier P, Mayer E: Pure representational neglect after right thalamic lesion. Annals of Neurology 2001, 50:401-404.

* 23 Zorzi M, Priftis K, Meneghello F, Marenzi R, Umilta C: The spatial representation of numerical and non-numerical sequences: Evidence from neglect. Neuropsychologia 2006, 44:1061-1067.

Neglect patients produced rightward-shifted medians of imagined numerical intervals, but not of nonnumerical intervals, such as those between letters or months.

24 Doricchi F, Guariglia P, Gasparini M, Tomaiuolo F: Dissociation between physical and mental number line bisection in right hemisphere brain damage. Nature Neuroscience 2005, 8:16631665.

25 Galton F: Visualised numerals. Nature 1880, 21:252-256.

* 26 Loetscher T, Brugger P: A disengagement deficit in representational space. Neuropsychologia 2007, 45:1299-1304.

New, ingenious tests were used in this single case study to explore the mechanisms of imaginal 
neglect.

27 Bartolomeo P, Urbanski M, Chokron S, Chainay H, Moroni C, Siéroff E, Belin C, Halligan P: Neglected attention in apparent spatial compression. Neuropsychologia 2004, 42:49-61.

* 28 Rode G, Revol P, Rossetti Y, Boisson D, Bartolomeo P: Looking while imagining: The influence of visual input on representational neglect. Neurology 2007, 68:432-437.

Patients with imaginal neglect produced incomplete descriptions of a map of France, irrespective of the presence or absence of (task-unrelated) visual stimuli.

** 29 Doricchi F, Iaria G, Silvetti M, Figliozzi F, Siegler I: The "ways" we look at dreams: evidence from unilateral spatial neglect (with an evolutionary account of dream bizarreness). Experimental Brain Research 2006 [Epub ahead of print].

An intriguing report of REM asymmetries in a neglect patient, and of their relation to his dreams.

30 Bartolomeo P: The relationship between visual perception and visual mental imagery: a reappraisal of the neuropsychological evidence. Cortex 2002, 38:357-378.

31 Binder J, Marshall R, Lazar R, Benjamin J, Mohr JP: Distinct syndromes of hemineglect. Archives of Neurology 1992, 49:1187-1194.

* 32 Siéroff E, Decaix C, Chokron S, Bartolomeo P: Impaired orienting of attention in left unilateral neglect: A componential analysis. Neuropsychology 2007, 21:94-113.

A systematic analysis of the component deficits of spatial orienting in 26 patients with right brain damage. The anatomical correlates of the deficits are also described, but only clinical images with limited resolution were available.

33 Bartolomeo P, Chokron S: Orienting of attention in left unilateral neglect. Neuroscience and Biobehavioral Reviews 2002, 26:217-234.

34 Bisiach E: Mental representation in unilateral neglect and related disorders. The Quarterly Journal of Experimental Psychology 1993, 46A:435-461.

* 35 Coulthard E, Parton A, Husain M: The modular architecture of the neglect syndrome: Implications for action control in visual neglect. Neuropsychologia 2007:doi:10.1016/j.neuropsychologia.2007.1001.1020.

A commentary stressing the notion of neglect as a multi-component syndrome. According to the authors, the precise combination of component deficits may vary between patients, and no single deficit needs to be crucial to neglect.

36 D'Erme P, Robertson IH, Bartolomeo P, Daniele A, Gainotti G: Early rightwards orienting of attention on simple reaction time performance in patients with left-sided neglect. Neuropsychologia 1992, 30:989-1000.

* 37 Natale E, Marzi CA, Bricolo E, Johannsen L, Karnath HO: Abnormally speeded saccades to ipsilesional targets in patients with spatial neglect. Neuropsychologia 2007, 45:263-272.

A carefully conducted study on saccadic reaction times in patients with left neglect, demonstrating faster saccades for targets appearing 5 and 10 degrees right of fixation.

38 Posner MI, Walker JA, Friedrich FJ, Rafal RD: Effects of parietal injury on covert orienting of attention. Journal of Neuroscience 1984, 4:1863-1874. 
39 Lupiáñez J, Decaix C, Siéroff E, Chokron S, Milliken B, Bartolomeo P: Independent effects of endogenous and exogenous spatial cueing: Inhibition of return at endogenously attended target locations. Experimental Brain Research 2004, 159:447-457.

** 40 Snow JC, Mattingley JB: Goal-driven selective attention in patients with right hemisphere lesions: how intact is the ipsilesional field? Brain 2006, 129:168-181.

Six patients with left neglect judged either the identity or the colour of centrally presented letters flanked by distractor letters, which shared one dimension (identity or colour) with the target. Left-sided flankers slowed RTs only in the task-relevant dimension, similar to controls' performance. In contrast, right-sided flankers slowed RTs independent of their relevance to the task. Thus, rightward attentional capture in these patients was not followed by normal, goaldriven attentional selection.

41 Robertson IH: Do we need the "lateral" in unilateral neglect? Spatially nonselective attention deficits in unilateral neglect and their implications for rehabilitation. NeuroImage 2001, 14:S85S90.

42 Husain M, Rorden C: Non-spatially lateralized mechanisms in hemispatial neglect. Nature Reviews Neuroscience 2003, 4:26-36.

43 Malhotra P, Jager HR, Parton A, Greenwood R, Playford ED, Brown MM, Driver J, Husain M: Spatial working memory capacity in unilateral neglect. Brain 2005, 128:424-435.

44 Bartolomeo P, Chokron S: Left unilateral neglect or right hyperattention? Neurology 1999, 53:20232027.

45 Bartolomeo P, Chokron S, Siéroff E: Facilitation instead of inhibition for repeated right-sided events in left neglect. NeuroReport 1999, 10:3353-3357.

46 Bartolomeo P: The novelty effect in recovered hemineglect. Cortex 1997, 33:323-332.

* 47 Habekost T, Rostrup E: Visual attention capacity after right hemisphere lesions. Neuropsychologia 2007, 45:1474-1488.

Patients with right hemisphere lesions identified letters presented in brief visual displays. Bilaterally impaired processing speed was related to injury to the right middle frontal gyrus, whereas storage capacity deficits correlated with severe and diffuse white matter damage of the frontoparietal connections.

48 Halligan PW, Fink GR, Marshall JC, Vallar G: Spatial cognition: evidence from visual neglect. Trends in Cognitive Sciences 2003, 7:125-133.

* 49 Committeri G, Pitzalis S, Galati G, Patria F, Pelle G, Sabatini U, Castriota-Scanderbeg A, Piccardi L, Guariglia C, Pizzamiglio L: Neural bases of personal and extrapersonal neglect in humans. Brain 2007, 130:431-441.

An anatomical study on 52 right brain-damaged patients. The authors concluded that personal neglect is due to lesion involvement of the supramarginal gyrus in the parietal lobe, whereas extrapersonal neglect results from damage of ventral areas including the STG and the inferior 
frontal gyrus. SLF involvement, which was present in both groups of patients, went unfortunately undiscussed.

50 Karnath $\mathrm{H}-\mathrm{O}$, Fruhmann Berger M, Kuker W, Rorden C: The anatomy of spatial neglect based on voxelwise statistical analysis: a study of 140 patients. Cerebral Cortex 2004, 14:1164-1172.

51 Karnath H-O, Ferber S, Himmelbach M: Spatial awareness is a function of the temporal not the posterior parietal lobe. Nature 2001, 411:950-963.

52 Hillis AE, Newhart M, Heidler J, Barker PB, Herskovits EH, Degaonkar M: Anatomy of spatial attention: insights from perfusion imaging and hemispatial neglect in acute stroke. Journal of Neuroscience 2005, 25:3161-3167.

53 Godefroy O, Duhamel A, Leclerc X, Saint Michel T, Henon H, Leys D: Brain-behaviour relationships. Some models and related statistical procedures for the study of brain-damaged patients. Brain 1998, 121 (Pt 8):1545-1556.

54 Bartolomeo P: A parieto-frontal network for spatial awareness in the right hemisphere of the human brain. Archives of Neurology 2006, 63:1238-1241.

A short review of fronto-parietal disconnection in left neglect, with a discussion of methodological issues concerning the limitations of lesion overlapping studies in stroke patients and of direct brain stimulation in neurosurgical patients.

55 Shindo K, Sugiyama K, Huabao L, Nishijima K, Kondo T, Izumi S: Long-term effect of low-frequency repetitive transcranial magnetic stimulation over the unaffected posterior parietal cortex in patients with unilateral spatial neglect. Journal of Rehabilitation Medicine 2006, 38:65-67.

56 Ellison A, Schindler I, Pattison LL, Milner AD: An exploration of the role of the superior temporal gyrus in visual search and spatial perception using TMS. Brain 2004, 127:2307-2315.

57 Corbetta M, Kincade MJ, Lewis C, Snyder AZ, Sapir A: Neural basis and recovery of spatial attention deficits in spatial neglect. Nature Neuroscience 2005, 8:1603-1610.

58 Thiebaut de Schotten M, Urbanski M, Duffau H, Volle E, Lévy R, Dubois B, Bartolomeo P: Direct evidence for a parietal-frontal pathway subserving spatial awareness in humans. Science 2005, 309:2226-2228.

* 59 Catani M: Diffusion tensor magnetic resonance imaging tractography in cognitive disorders. Current Opinion in Neurology 2006, 19:599-606.

A balanced presentation of the advantages and limitations of the use of DT-MRI tractography in cognitive neuroscience.

** 60 Schmahmann JD, Pandya DN: Fiber Pathways of the Brain. New York: Oxford University Press; 2006.

The reference atlas of the organization of white matter fibre pathways in the rhesus monkey.

61 Thiebaut de Schotten M, Kinkingnéhun SR, Delmaire C, Lévy R, Volle $E$, Dubois B, Lehéricy S, Duffau $\mathrm{H}$, Bartolomeo P: OVER-TRACK: A new tool to visualize the white matter organization in Talairach space. Neuroimage 2006, 31:S110. 
** 62 Bartolomeo P, Thiebaut de Schotten M, Doricchi F: Left unilateral neglect as a disconnection syndrome. Cerebral Cortex 2007:doi: 10.1093/cercor/bhl1181.

A review of recent advances on the anatomical bases of neglect, suggesting that intra- and interhemispheric disconnection disrupting large-scale brain networks may be a more likely correlate of neglect than dysfunction of single cortical regions.

63 Gharabaghi A, Fruhmann Berger M, Tatagiba M, Karnath HO: The role of the right superior temporal gyrus in visual search-insights from intraoperative electrical stimulation. Neuropsychologia 2006, 44:2578-2581.

64 Milner B: Visual recognition and recall after right temporal-lobe excision in man. Epilepsy \& Behavior 2003, 4:799-812.

* 65 Di Ferdinando A, Parisi D, Bartolomeo P: Modeling orienting behavior and extinction with "ecological" neural networks. Journal of Cognitive Neuroscience 2007.

Computer-simulated agents learned to perform a discrimination task by self-organizing their internal connections. Agents learned to orient their eye toward a peripherally presented object, thus demonstrating the emergence of orienting behaviour. Partial network disconnection determined impaired orienting, which led to an analogue of extinction/neglect.

66 Mesulam MM: Spatial attention and neglect: parietal, frontal and cingulate contributions to the mental representation and attentional targeting of salient extrapersonal events. Philosophical Transactions of the Royal Society of London B 1999, 354:1325-1346.

* 67 Park KC, Lee BH, Kim EJ, Shin MH, Choi KM, Yoon SS, Kwon SU, Chung CS, Lee KH, Heilman KM, et al.: Deafferentation-disconnection neglect induced by posterior cerebral artery infarction. Neurology 2006, 66:56-61.

In this study on 45 patients with posterior cerebral artery infarctions, neglect was found to correlate with occipital damage together with complete injury to the splenium of the corpus callosum.

* 68 Bird CM, Malhotra P, Parton A, Coulthard E, Rushworth MF, Husain M: Visual neglect following right posterior cerebral artery infarction. Journal of Neurology, Neurosurgery and Psychiatry 2006, 77:1008-1012.

This study on patients with infarcts in the territory of the right posterior cerebral artery indicated a possible disconnective correlate of neglect in white matter tracts running in the depth of the temporal lobe. 


\section{Figure legends}

Fig. 1. Performance of a patient with left neglect two months after an ischemic lesion in the territory of the right anterior choroidal artery, when drawing a butterfly from memory, first with (upper panel), and then without (lower panel) visual guidance (while blindfolded), whereupon left neglect disappeared (reprinted from Ref. [19], with the authors' permission).

Fig. 2. Three long-range fronto-caudal white matter pathways in the right hemisphere of the normal human brain, with their cortical projections [61]. The arcuate fasciculus (red) and the human homologues of the second (yellow) and third (green) branches of the superior longitudinal fasciculus are shown. 